\title{
PRE-POST OBSERVATION RESEARCH FOSTERS A PRELIMINARY STUDY IN PRODUCT FORM IDENTITY
}

\author{
Mohd Hasni CHUMIRAN ${ }^{1}$, Shahriman Zainal ABIDIN ${ }^{1}$ and Muhammad Jameel Mohamed \\ KAMIL $^{2}$ \\ ${ }^{1}$ Department of Industrial Design, Universiti Teknologi MARA (UiTM), Malaysia \\ ${ }^{2}$ School of the Arts, Universiti Sains Malaysia (USM), Malaysia
}

\begin{abstract}
This research was specifically developed for the preliminary study of verbal protocol analysis (VPA) for the recognition of design representation underlying a design activity phenomenally based on design research ethics. The ethics case study purposely aims to develop the pre-post observation on a rigorous prescriptive study of the think-aloud design experience according to the eco-design identity in the product form context. Eight pilot participants were sampled from the degree-level product design programme from the School of Arts, Universiti Sains Malaysia, Penang, Malaysia. In the preobservational study, the emphasis on design pattern via a reflective practice (RP) approach using the visual imagery interactions between interpolative and extrapolative strategies was utilised by design scheme method. Meanwhile in the post-observational analysis, rubrics for evaluation were used as a design tool assessment to evaluate the concept of eco-design artefact by using the quality of the morphological patterns. The rehearsal prescriptive analysis, also known as s VPA's preliminary study (VPAPS), was predicted to show the design representation (by contextual learning) with the interpretation of the eco-design identity. The outcome should present an encode of the designer's human cognition framed by the design phenomena's existence within an educational reliability test. By using dual method triangulation, a rigorous VPA method ethically achieved a future design criterion as the goal of design representation pertaining to real-fieldwork case study for design and engineering education.
\end{abstract}

Keywords: Design education, ethics, preliminary study, form, verbal protocol analysis

\section{INTRODUCTION}

A design phenomenon is vital for every design activity, especially for the industrial designers who derive their design prediction based on the design information needs [1], [2], [3]. The inquiry of design phenomenon normally occurs when a product design fashion encounters a beyond continuum of design movement dependency which builds diverse design identities surrounding society and the environment ecologically ${ }^{1}$.
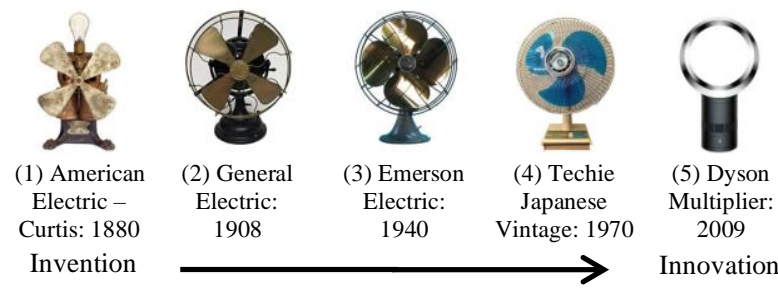

Electric:

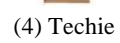

Japanese

Japanese Multiplier:

1908

2009

\footnotetext{
${ }^{1}$ Extrapolative morphing concept sources:

(1) https://americanhistory.si.edu/lighting/c_choice/choice_3a.htm

(2) https://earlyfans.blogspot.my/2011/02/ge-pancake-1894-1908.html

(3) https://www.fancollectors.org/Emerson2/emerson2.htm

(4) https://www.etsy.com/listing/159003739/techie-japanese-vintage-electric-fan?show_sold_out_detail=1

(5) https://www.theguardian.com/technology/2014/jul/18/dyson-cool-am06-review-desk-fan.
} 
Figure 1. Beyond continuum extrapolative strategy - morphing (Non-linear/non-uniform)

Figure 1 illustrates the role of the beyond-artefact's influence on the extrapolative morphing concept. This morphing concept relates to evolution of form (EoF) which refers to the non-linear product-form development, known as hermeneutical intangible ecological form [1], [3]. Interpreting the design context, the visual imagery influences two key features of the design phenomena that are patterned according to (1) the morphological design and (2) the visual elements. Emphasis is on the VPA via the visual imagery of the design representation in that that the think-aloud of the design experience could encode a design prediction and explores the participants thought process. Additionally, it also explores the participation thought process.

The ethics case study that is observed is therefore empirically defined as a design prediction through design experience activities [1], [2]. Accordingly, this VPA-based method imposes a rigorous preliminary study following two research questions: (1) what kind of design sketch criterion is required from the drawing process? And (2) what method of design sketch develops the design theme and product form identity?

\section{PROCEDURE OF THE VPA'S PRELIMINARY STUDY}

The rehearsal conception of VPAPS is to identify the product form identity within the essence of scientific reasoning itself. This validation of verbalize aloud address the design representation through an artificial intelligence (AI) platform using the designer's behaviour upon drawing activities from the real world of design experiences [3]. For the design classification, the study uses the axiom approach to manage the number of design theme families that it gathered from the prior literature review and the descriptive study phase. The axiom approach usually catalyses a complex phenomenon by using the dimension of branches and conceptual domains concurrently [1]. Hence, the VPA method inquiry must deliver some proper ethical practices before entering the real-fieldwork case study.

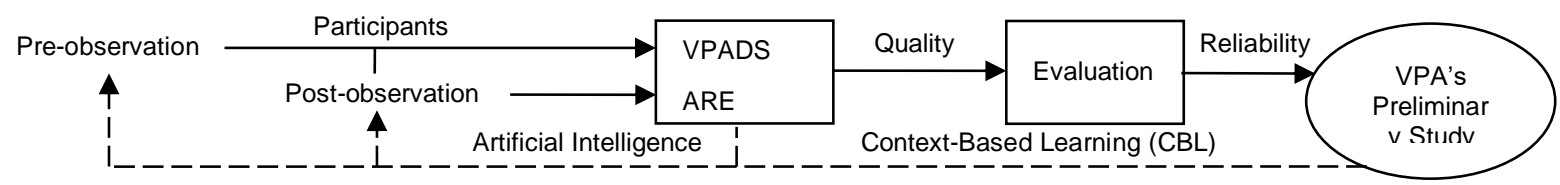

Figure 2. The flow process for VPA's preliminary study

To understand this process, contextual learning is required. Two properties of observation are focused on the end-to-end procedure review as shown in Figure 2. Three ethical ambiguities encompass the entire VPAPS process, as explained below:

\subsection{Pre-observation: verbal protocol analysis and equipment}

The purpose of the drawing practice (activities) is to identify the specific design-thinking for the design evolution of the product form. This is done with sufficient time for completion. Goals are defined by the observer for the participants to achieve and an approximate timeframe for the manual design scheme is to be estimated.

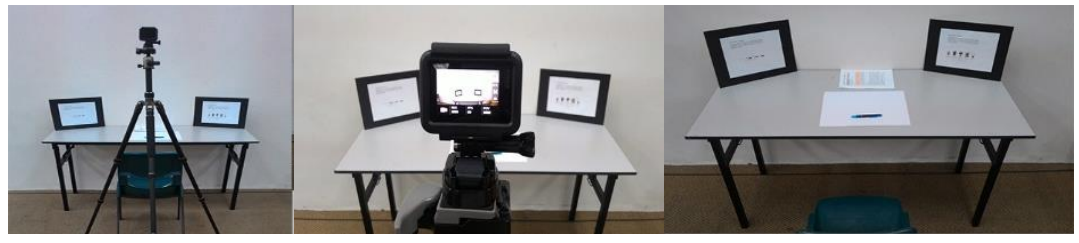

Figure 3. The VPA method preparation

Six steps of pre-observation are required for the VPA transcription study. A VPA's essential needs are: (1) demographic information, (2) design theme briefing and VPA information, (3) visual imagery interactions i.e.: mood board (visual aid) and reading material, (4) on/off site mode and camera layout setup, (5) drawing media and instrument, and a (6) proposed estimated time. At this stage, there are no participants involved in the process (see Figure 3). The VPA process uses a manual design scheme, which means a timeframe illustration for data analysis together with the transcription stage. Secondly, it effectively maps out the design path and follows the supply chain. At this point, the study will define the design theme according to the five VPA disciplines, namely (1) cognition, (2) recognition, (3) 
iteration, (4) comprehension, and (5) association. These VPA disciplines come from the reflective practice model developed by Schon's theory of practice [1].

\subsection{Post-observation: output materials and design tools' assessment}

Post-observation refers to the drawing and/or design sessions whereby the time of the design activity (demonstration) encourages the participants to verbalize aloud and focus on their drawing skill. They also develop their design process educationally to produce the design manuscript as output content.

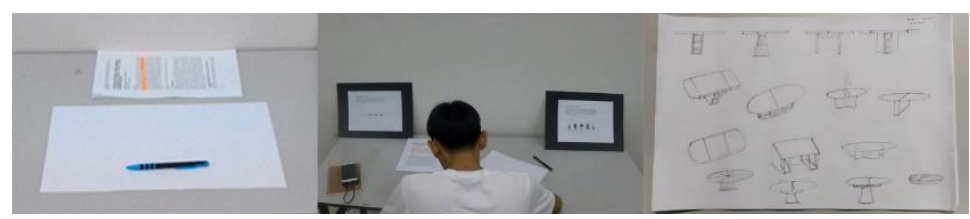

Figure 4. Instruments: media; videotaping: record; design manuscript: output VPA process

In Figure 4, three design activities set the VPA process stages. These assist in improving the content of the design manuscript and capture the essence of its morphologically. Using this approach called the contextual learning, the participant shares design experiences such as (1) the basic instruments used, (2) provides a pattern of design process demonstration, and (3) is encouraged to do RP in order to encode a think-aloud of the drawing content within the participant's behavioural capability. This is known as the design cognition area. Consequently, two data points are required in the post-observation, namely (1) a design manuscript and (2) video recording at a HD resolution of $1080 \mathrm{p}(1920 \times 1080)$.

\subsection{Evaluation of the pre-post observation research}

The VPA strategy analysis uses a manual form of design scheme transcription. The case study expert emphasised that manual practice would shape the quality and enhance the reality of grounded research naturally [1], [3], [5].

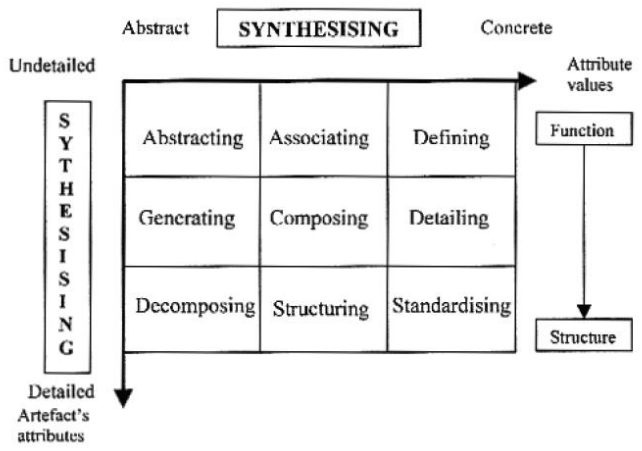

Figure 5. Taxonomy of design definition activities [7]

A manual transcription strategy properly channels the pattern of message communication by catalysing and screening the characteristics of "encodes" or "decodes" according to the design belief [8]. This VPAPS emphasises the experience model of Mono (1997) in that the product interaction process allows designers to discover the visual imagery of the design communication digitally. In Figure 5, the prepost observation evaluation triangulates on the design attributes. The taxonomy of design definitions could then be used to diagnose similarities in answers via the diagonal mode: (1) abstracting, (2) composing, and (3) standardising. Pre-observation looks at the transcription of the manual design scheme and computes the best way for participants to accomplish their tasks. Post-observation comments on the direction of the method process and how the manuscripts used in evidence-based computing can be proven. Thus, an ethics case study can simultaneously screen the designers' thought process and design philosophy.

\section{METHOD AND DATA ANALYSIS}

Purposeful sampling was used in the pilot study. This non-probability sampling represents small homogeneous characteristics, which allows the researcher to describe a subgroup and avoid generalisation. In a grounded theory study, the qualitative experts suggest that a small sample size should consist of four to forty-two $(n=4<42)$ participants [1], [5]. Hence, eight respondents from an 
Accelerated Programme for Excellence (APEX) university status - granted by Malaysian government should possibly represent the Malaysian universities. The participants interviewed were from the final year of degree-level product design programme from the School of Arts, Universiti Sains Malaysia, Penang, Malaysia. Realistically, 22 other institutions, including government and private universities, offer product design and/or industrial design programmes recognized by Malaysian Qualifications Register (MQR) which are under The Malaysian Qualifications Agency (a statutory body governed by the Ministry of Education). This signifies that other Malaysian institutions pattern the same area of design education programmes. This saturation of sampling size is an appropriate number for purposeful sampling in a focus group with a small sample size. Therefore, the small focus group in this study was divided into two groups, namely group A and group B. The study emphasises two approaches: (1) VPA design scheme method and (2) analytic rubrics evaluation.

\subsection{Development of the VPA design scheme method}

In Figure 6, the manual design scheme is a visual aid graph analysis, the time and think-aloud recorder displays the duration and determines whether the length of the experiment is sufficient or otherwise [1], [6], [7]. Implicitly, it represents the goal of that data on the design activities are gathered using a numerical scale for the design evaluation stage. Using interpolative and extrapolative strategies, the VPA design scheme (VPADS) method will compare the visual imagery of both interactions. This is achieved in the video recording session by transcribing the pre-observation.

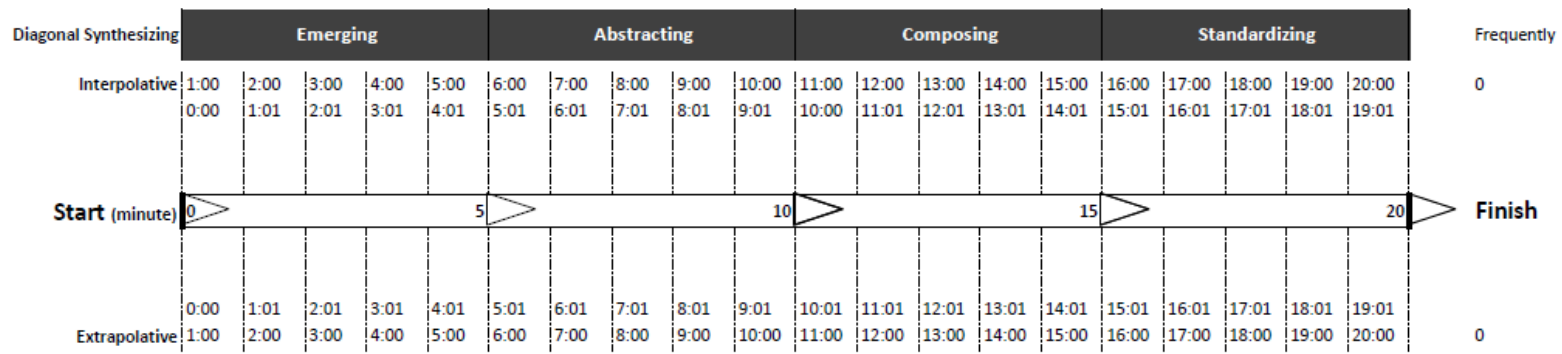

Figure 6. The method of the VPA design scheme manually using a comparison approach

The VPADS method begins with the Emerging phase, followed by another three phases of the diagonal synthesising process, namely Abstracting, Composing, and Standardising [7]. It takes five minutes respectively and the whole process must be accomplished within 20 minutes. For the RP, the interpolative strategy in the upper section is against the extrapolative strategy in the lower section, where a comparison of the number of minutes is recorded throughout the session. The total minutes are shown at the Finish point.

\subsection{VPA grading of the analytic rubric's evaluation}

The analytic rubric evaluation (ARE) is the most efficient qualitative assessment tool for the various criteria in extracting the output of the think-aloud. This ARE interpreting the design manuscript to gather the data collection [4]. Here, two data points are developed, which are (1) items within the scoring scale and (2) indicative level of the participants' proficiency. The five specific criteria should be identified, and the scoring scale should be levelled using a 5-rank or semantics value. These criteria are established using the five VPA disciplines previously outlined. Ascending numerical values are required, which are analysed using a reliability test. Secondly, the indicative level of the participants' proficiency will be slotted-in together with the internal consistency of a subject's involvement. This could be rated using an ordinal item. However, the two data points can only be accepted if the Cronbach's Alpha value is not less than .70 , following the rule of thumb for the reliability coefficient.

\section{Discussion}

This ethics case study saw of the design phenomenon revealed by the participants' essence using the designer's ways of design thinking [8]. It also reflects the design methodology by means of an appropriate protocol to be used to understand design thinking among designers [7]. Hence, the discussion of the pre-post observation research based on Group A and Group B is as follows:

\subsection{Pre-observation: the design reflective practice encoding ethics case study for}




\section{product design in higher education}

In the VPADS method, the entire experimental preparation closely deals with the RP approach inspired by Schon's theory of practice. It was set up by transcribing the video recordings, as shown in Figure 5 earlier. First, the transcribed VPADS results showed that $50 \%$ of the participants had a good proficiency level, where four participants obtained a grading percentage of $80 \%$. Meanwhile, two participants had a very good proficiency level and another two participants obtained an excellent proficiency level, which represent the lower $25 \%$ of the total of frequency, respectively. Due to the mystery of design essence, the visual imagery interactions may expose the quality of the design manuscript that encodes the "cantilever principle pattern" during the video recording phase. For instance, qualitative reasoning is explicitly projected on the semantic patterns that the product's EoF conveys and the beyond-continuum understanding: the initial design of the American Electric (Curtis, 1880) morphed into the modern innovative design of the Dyson Multiplier (2009). Here, the Dyson desk fan product truly evoked the eco-design identity in the product form context. Secondly, using the analogical exercise, all these experiments morphed their patterns and semantics by using the extrapolative strategy (non-linear/nonuniform) along the video recording sessions simultaneously [1], [3], [6], [7], [8]. This empirical output sorts the design criteria so that the consistency emerges and is part of the design contingency. Therefore, a reliability test is required to examine evidence-based design as to display the numerical data interpretation.

\subsection{Post-observation: design manuscript footprint measures the think aloud interpretation for the engineering design (ED) field}

The footprint of the design manuscript derives the semantics of design thinking. This understanding hermeneutically interprets the quality of think-aloud among engineers' cognition. The quality of thinkaloud engages with the thought process as their natural logical algorithm and design system thought processes are in the ED field [1], [4].

Table 1. Reliability test and scale statistics

\begin{tabular}{|c|c|c|c|c|c|c|}
\hline Respondent & $\begin{array}{c}\text { Cronbach's } \\
\text { Alpha }\end{array}$ & $\begin{array}{c}\text { Cronbach's Alpha Based } \\
\text { on Standardised Items }\end{array}$ & Mean & Variance & $\begin{array}{c}\text { Std. } \\
\text { Deviation }\end{array}$ & $\begin{array}{c}\mathrm{N} \\
\text { of Items }\end{array}$ \\
\hline Group A & .778 & .767 & 22.5000 & 12.333 & 3.51188 & 6 \\
Group B & .937 & .942 & 23.2500 & 15.583 & 3.94757 & 6 \\
\hline
\end{tabular}

Hence, as shown in Table 1, the reliability test was conducted, and the results indicate that Group B is the best choice even though Group A's Cronbach's Alpha value also exceeds .70. Furthermore, the young designers' competence in drawing and design skills can be predicted beyond the product developed from the design manuscript. At this point, the ARE tool is required to measure the quality of the design manuscript using the scoring distinctions [4]. For instance, the study assigned five criteria items representing the design theme category, which are (1) cognition: drawing practice, (2) recognition: drawing quality, (3) iteration: visual elements, (4) comprehension: design pattern, and (5) association: design semantics. The findings showed that design pattern is correlated with most of the other criteria, which are drawing practice, drawing quality, and design semantics in the table for inter-item correlation matrix.

Table 2. Item-total statistics

\begin{tabular}{|c|c|c|c|c|c|}
\hline $\begin{array}{c}\text { Respondent } \\
\text { Group B }\end{array}$ & $\begin{array}{c}\text { Scale Mean } \\
\text { If Item } \\
\text { Deleted }\end{array}$ & $\begin{array}{c}\text { Scale Variance } \\
\text { If Item Deleted }\end{array}$ & $\begin{array}{c}\text { Corrected } \\
\text { Item-Total } \\
\text { Correlation }\end{array}$ & $\begin{array}{c}\text { Square } \\
\text { Multiple } \\
\text { Correlation }\end{array}$ & $\begin{array}{c}\text { Cronbach's } \\
\text { Alpha If Item } \\
\text { Deleted }\end{array}$ \\
\hline Design Pattern & 19.5000 & 9.000 & .986 & - & .903 \\
\hline
\end{tabular}

It consistently delivered the item-total statistics (see Table 2), which exceeds .30 and with an almost perfect correlation of $r=1.0$ in the statistical analysis. However, there is no correlation between drawing pattern and visual elements. 


\section{CONCLUSIONS}

In this study, two groups were screened, and Group B was selected as it scored significantly higher and better achieved the contingency design representation for answering the two research questions earlier. The results revealed that the drawing pattern criteria is highly recommended under the roles of dual method triangulation; the VPADS and ARE method. For validity and reliability, these criteria are evaluated and tested using the qualitative assessment tool within the consistency reliability measurement respectively. The RP approach as a contextual learning methodology was embodied in the semantics of eco-design identity. Therefore, the research impact which addresses the VPAPS following the ethics case study (1) may possibly predict other futures of the product form and identity, (2) the CBL understanding as a digitization tool for the descriptive study and (3) improving the designer's sensory imagery in design information ecologically. Hence, this study is pedagogically important in bridging two domains between ID and ED fields and to comprehend a designer's thought process with humancentred design orientation, especially empowering a new product development in design education.

\section{ACKNOWLEDGEMENT}

This research is gratefully supported by Universiti Teknologi MARA [Grant number: 600-IRMI/FRGS 5/3 (425/2019)] and Ministry of Education Malaysia under the FRGS Scheme [Sponsorship File no: FRGS / 1 / 2019 / SS107 / UiTM / 02 / 4].

\section{REFERENCES}

[1] Abidin, S. Z. Practice-based Design Thinking for Form Development and Detailing, Norwegian University of Science and Technology, 2012:270, Trondheim, 2012.

[2] Institut Dominicain d'Etudes Orientales (IDEO), The Little Book of Design Research Ethics. $1^{\text {st }}$ Edition. Available: https://www.ideo.com/post/the-little-book-of-design-research-ethics [Accessed on 2017, 22 September], (2016) February.

[3] Kelley, T. R. Capobianco, B. M. and Kaluf, K. J. Concurrent Think-aloud Protocols to Assess Elementary Design Students, International Journal of Technology and Design Education, 25(4), 2014, pp. 521-540.

[4] Jonsson, A. and Svingby, G. The use of scoring rubrics: Reliability, validity and educational consequences. Educational Research Review, 2 (2), 2007, pp.130-144.

[5] Vasileiou, K., Barnett, J., Thorpe, S., \& Young, T. Characterising and justifying sample size sufficiency in interview-based studies: systematic analysis of qualitative health research over a 15-year period. BMC Medical Research Methodology, 2018, pp. 18(1).

[6] Cowan, J. The Potential of Cognitive Think-aloud Protocols for Educational Action-Research. Active Learning in Higher Education, Musselburgh, 2017, pp.1-14.

[7] Zainal Abidin, S. Christoforidou, D. and Liem, A. Thinking and Re-Thinking Verbal Protocol Analysis in Design Research, the 17th International Conference on Engineering Design (ICED 09), DS 58-2, Vol. 2, , California, 2009, pp. 1-12.

[8] Jamaludin, M. S. Zulkapli, M. F. and Zainal Abidin, S. The Characteristics of Form in Relation to Product Emotion, the 15th International Conference on E\&PDE 2013, DS 76, Dublin, 2013, pp. 716-721. 